
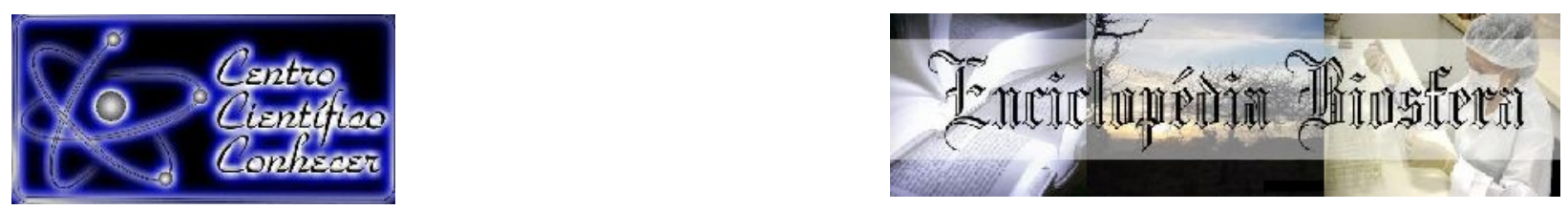

\title{
CUIDADO INTEGRAL AOS PACIENTES COM CÂNCER NAS REDES DE ATENÇÃO À SAÚDE
}

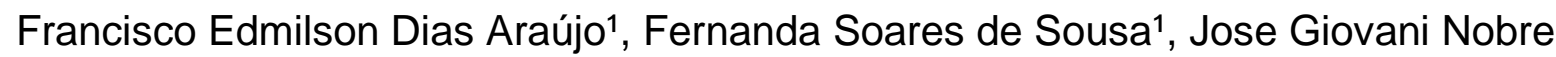
Gomes², Sara Taciana Firmino Bezerra², Themis Cristina Mesquita Soares² $^{2}$

1 Discente do Programa de Pós-Graduação em Planejamento e Dinâmicas Territoriais no Semiárido (PLANDITES) da Universidade do Estado do Rio Grande do Norte - UERN - e-mail: franciscoedmilson@alu.uern.br

2 Docente do Programa de Pós-Graduação em Planejamento e Dinâmicas Territoriais no Semiárido (PLANDITES) da Universidade do Estado do Rio Grande do Norte - UERN

Recebido em: 15/11/2021 - Aprovado em: 15/12/2021 - Publicado em: 30/12/2021

DOI: 10.18677/EnciBio 2021D21

trabalho licenciado sob licença Creative Commons Attribution-NonCommercial-NoDerivatives 4.0 International License

\section{RESUMO}

Trata-se de um estudo com o objetivo de compreender o desenvolvimento do cuidado ao paciente com câncer nas redes de atenção à saúde, por meio de uma revisão integrativa de literatura. Procedeu-se com a coleta de dados a partir do Portal Regional da Biblioteca Virtual em Saúde (BVS), indexado pela BIREME. Ao serem aplicados os critérios de exclusão, foram analisados 10 artigos, oriundos das bases LILACS, BDENF e MEDLINE. Observou-se que câncer de mama é o assunto mais presente nos estudos e que o apoio da família, dos profissionais e dos grupos sociais são importantes para a recuperação da paciente. Identificou-se que o processo de trabalho ainda apresenta algumas fragilidades, sobretudo no que diz respeito à regulação e ao acolhimento. Evidenciaram-se fatores que estão relacionados às deficiências na gestão da rede de atenção à saúde e à ausência de uma rede de apoio efetiva.

PALAVRAS-CHAVE: Continuidade da assistência; Modelos de Assistência à Saúde; Neoplasias.

\section{INTEGRAL CARE FOR PATIENTS WITH CANCER IN THE HEALTH CARE NETWORKS: AN INTEGRATIVE REVIEW}

\begin{abstract}
This is a study aimed at understanding the development of care for cancer patients in health care networks, through an integrative literature review. This study proceeded with the collection of data from the Regional Portal of the Biblioteca Virtual em Saúde (BVS), indexed by BIREME. When applying the exclusion criteria, ten articles it is be analyzed, from the LILACS, BDENF and MEDLINE databases. It was observed that breast cancer is the most common subject in studies and that the support of family, professionals and social groups are important for the patient's recovery. It has been identified that the work process still has some weaknesses, especially with regard to regulation and reception. Factors that are related to deficiencies in the management of the health care network and the absence of an effective support network were evidenced.
\end{abstract}

KEYWORDS: Continuity of Care. Health Care Models; Neoplasms; 


\section{INTRODUÇÃO}

Dados do Ministério da Saúde (BRASIL, 2019) apontam que as doenças crônicas não transmissíveis (DCNT) representam um problema de saúde pública preocupante no cenário mundial, sendo um grupo de doenças de maior magnitude e que atinge principalmente as populações mais vulneráveis (como as de média e baixa renda e escolaridade), podendo ser atribuído a isso o acesso restrito às informações e aos serviços de saúde.

No ano de 2016,76,4\% das mortes gerais no Brasil foram decorrentes de doenças não transmissíveis, sendo que este quantitativo representa $83,3 \%$ das mortes do sexo feminino e $71,1 \%$ das mortes do sexo masculino. Nesse mesmo contexto, as neoplasias estão entre as principais causas de morte prematura na população brasileira em ambos os sexos, sobretudo na faixa etária de 30 a 69 anos (BRASIL, 2019).

Para o triênio de 2020-2022, estima-se uma incidência de 309.230 novos casos de neoplasias malignas a cada ano no Brasil, sendo os mais prevalentes: próstata (homens); mama e colo do útero (mulheres); e traqueia, brônquio e pulmão, cólon e reto, estômago e cavidade oral para ambos os sexos (INCA, 2019), tornando esse problema uma prioridade nacional na área de saúde pública.

A vigilância às DCNT se desenvolve por meio de três componentes: monitoramento dos fatores de risco; monitoramento da morbidade e mortalidade específica das doenças; e as respostas dos sistemas de saúde (WHO, 2011), de modo que neste último componente estão inclusos os elementos da gestão (políticas, planos, infraestrutura de apoio), profissionais e, sobretudo, o acesso a serviços essenciais como medicamento e exames complementares.

O Plano de Ações Estratégicas para o enfrentamento das DCNT no Brasil 2011-2022 (BRASIL, 2011) fundamenta-se em três eixos: (a) vigilância, informação, avaliação e monitoramento; (b) promoção da saúde; e (c) cuidado integral. Este último eixo inclui ações como: Linha de cuidado de DCNT, capacitação e telemedicina, medicamentos gratuitos e garantia de continuidade de cuidados, sendo essas ações integradas às Redes de Atenção à Saúde (RASs).

As RASs são arranjos produtivos híbridos que combinam a concentração de certos serviços com a dispersão de outros, devendo estruturar-se com base nos seguintes fundamentos: economia de escala, disponibilidade de recursos, qualidade e acesso; integração horizontal e vertical; processos de substituição; territórios sanitários; e níveis de atenção (MENDES, 2011). As Redes de Atenção integram e organizam os sistemas de atenção à saúde, compostos articulados para responder às necessidades, demandas e preferências das sociedades.

No caso da Rede de Atenção às Pessoas com Doenças Crônicas (RAPCDC), a implantação objetiva promover mudanças no modelo de atenção à saúde, a partir de uma série de dimensões, tais como: acolhimento; atenção centrada na pessoa e na família; cuidado continuado/atenção programada; atenção multiprofissional; projeto terapêutico singular; regulação da rede de atenção; apoio matricial; acompanhamento não-presencial; atendimento coletivo; autocuidado; linhas de cuidado e diretrizes clínicas; estratificação de risco; e educação permanente (BRASIL, 2013).

Assim, objetivou-se com o presente estudo compreender o desenvolvimento do cuidado ao paciente com câncer nas redes de atenção à saúde, por meio de uma revisão integrativa de literatura. 


\section{DESENVOLVIMENTO}

Para a elaboração da questão de pesquisa norteadora deste estudo, apropriou-se da estratégia conhecida como PVO (População, Variáveis e Resultado - outcomes). Nesse sentido, delimitou-se como direcionamento: Como estão estruturadas as ações de cuidado ao paciente com câncer nas redes de atenção à saúde?

Assim, desenvolveu-se uma revisão integrativa de literatura, de modo a sintetizar e avaliar os resultados de outras pesquisas sobre a temática. A pesquisa foi realizada no mês de maio de 2021 a partir do Portal Regional da Biblioteca Virtual em Saúde - BVS, parte integrante da Biblioteca Virtual em Saúde para América Latina e Caribe (bvsalud.org) indexado pela BIREME.

Utilizaram-se como termos da pesquisa "rede" e "cancer" (para a variação câncer não houve alteração nos resultados), tendo como indicador booleano "AND" e como critério "Title" (incluir as pesquisas que apresentam ambas as palavras no título). Utilizaram-se inicialmente como critérios de inclusão possuir texto completo disponível e publicados nos últimos cinco anos (2015/2020), obtendo os resultados conforme apresentado na Figura 01:

FIGURA 1 - Filtros iniciais utilizados na pesquisa

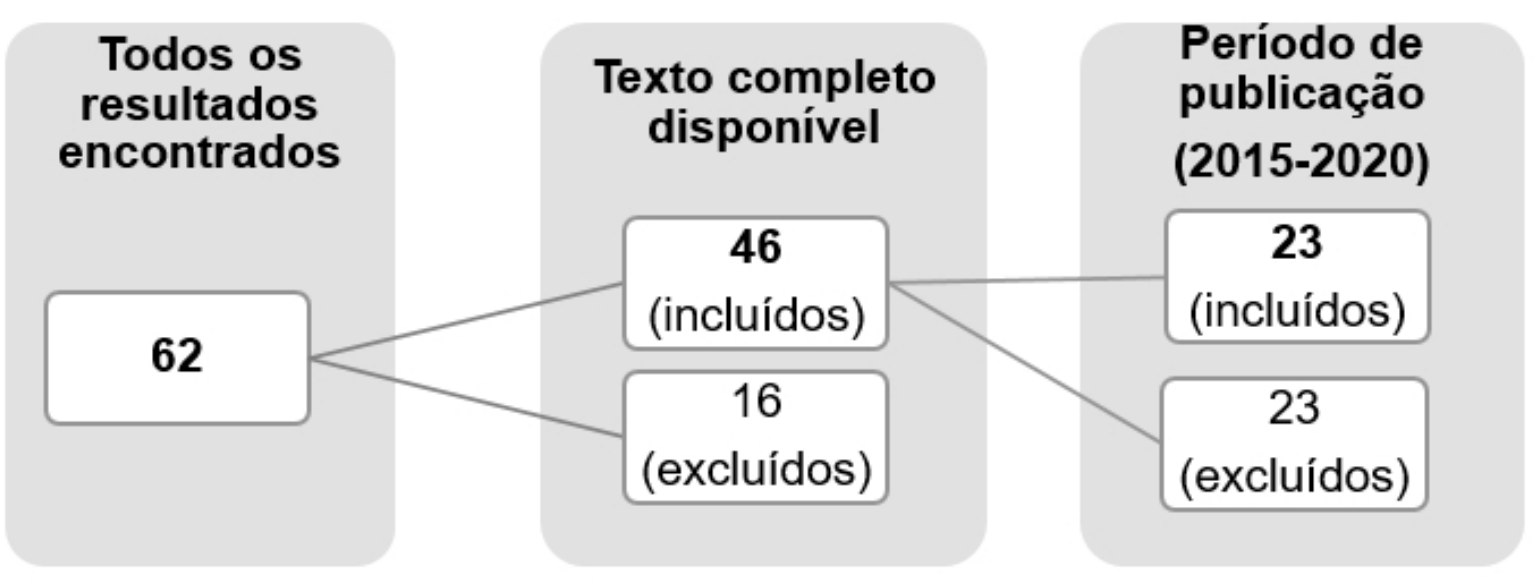

Fonte: os autores (2021)

Os artigos incluídos na primeira seleção estão disponíveis em três bases de dados, conforme distribuição apresentada na Tabela 1:

TABELA 1 - Distribuição dos documentos encontrados na pesquisa segundo Base de Dados no período de 2015 a 2020.

\begin{tabular}{|c|c|}
\hline Base de Dados & Quantidade \\
\hline LILACS & 15 \\
\hline BDENF - Enfermagem & 06 \\
\hline MEDLINE & 02 \\
\hline Total & 23 \\
\hline
\end{tabular}

Fonte: os autores (2021)

Em seguida, utilizou-se como filtro de seleção a leitura de todos os títulos para identificar os estudos duplicados, obtendo-se 17 documentos únicos (sem repetições). Logo após, foi realizada a leitura de todos os resumos, aplicando os seguintes critérios de inclusão e exclusão: 
FIGURA 2 - Seleção da amostra a partir dos critérios de inclusão e exclusão

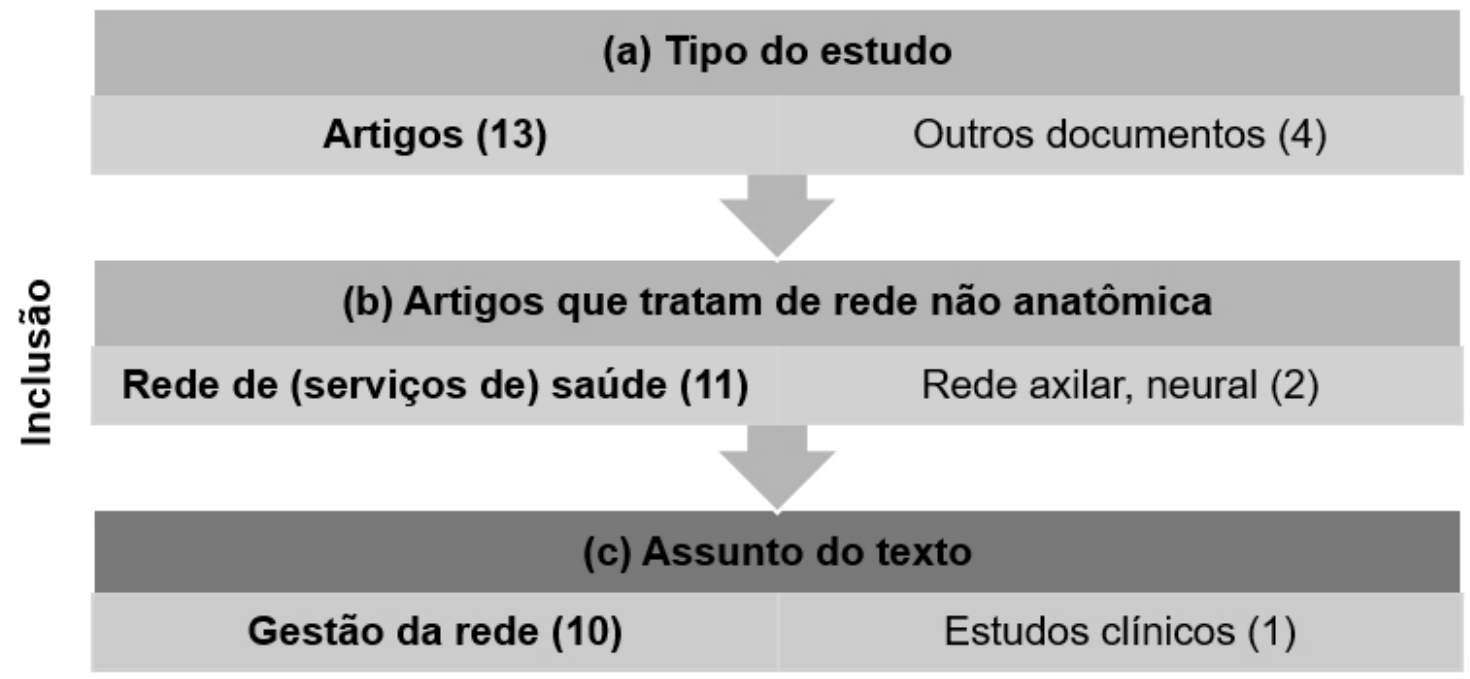

Fonte: os autores (2021)

Os 10 artigos selecionados para a análise foram todos estudos primários, analisados a partir de duas dimensões: a caracterização dos estudos e a análise do funcionamento da rede apresentada. Os resultados dessa análise são apresentados a seguir.

\section{Caracterização dos estudos}

Inicialmente, procedeu-se com uma caracterização dos estudos, conforme apresentado no Quadro 1, a seguir:

QUADRO 1 - Caracterização dos estudos

\begin{tabular}{|c|c|c|c|}
\hline N.․․ & $\begin{array}{l}\text { ISE } \\
\mathrm{ES}\end{array}$ & $\begin{array}{l}\text { S I } \\
\text { is }\end{array}$ & $\begin{array}{l}\text { PRINCIPAIS RESULTADOS } \\
\text { / CONCLUSÕES }\end{array}$ \\
\hline E1 & 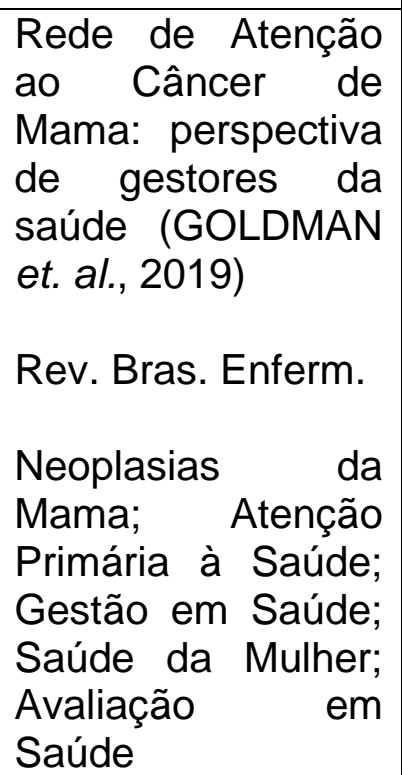 & $\begin{array}{l}\text { Analisar a organização } \\
\text { da Rede de Atenção } \\
\text { ao Câncer de Mama, } \\
\text { segundo a perspectiva } \\
\text { de gestores da } \\
\text { Coordenadoria } \\
\text { Regional de Saúde } \\
\text { Sudeste do município } \\
\text { de São Paulo. } \\
\text { Estudo descritivo, com } \\
\text { abordagem qualitativa. }\end{array}$ & $\begin{array}{l}\text { Há heterogeneidade na } \\
\text { operacionalização da Rede } \\
\text { de Atenção e os obstáculos } \\
\text { para sua integração e } \\
\text { integralidade foram } \\
\text { fragilidade do vínculo com as } \\
\text { usuárias; alta rotatividade de } \\
\text { profissionais na Atenção } \\
\text { Primária à Saúde; ausência } \\
\text { de padronização do sistema } \\
\text { de refência e e } \\
\text { contrarreferência, e baixa } \\
\text { cooperação entre serviços de } \\
\text { saúde municipais e } \\
\text { estaduais. }\end{array}$ \\
\hline $\mathrm{E}$ & $\begin{array}{l}\text { icer } \\
\text { vork: Struc } \\
\text { lysis of ena } \\
\text { ices / }\end{array}$ & $\begin{array}{l}\text { Estudo da rede } \\
\text { oncológica do Sistema } \\
\text { Único de Saúde no } \\
\text { estado de São Paulo }\end{array}$ & $\begin{array}{l}\text { estrutura e tamanho } \\
\text { suficiente para atender à } \\
\text { demanda de casos novos de }\end{array}$ \\
\hline
\end{tabular}




\begin{tabular}{|c|c|c|c|}
\hline N.음 & $\begin{array}{l}\text { REFERÊNCIAS E } \\
\text { DESCRITORES }\end{array}$ & $\begin{array}{l}\text { OBJETIVOS / } \\
\text { MÉTODOS }\end{array}$ & $\begin{array}{c}\text { PRINCIPAIS RESULTADOS } \\
\text { / CONCLUSÕES }\end{array}$ \\
\hline & $\begin{array}{l}\text { Rede de atenção } \\
\text { oncológica: análise } \\
\text { da estrutura de } \\
\text { serviços habilitados } \\
\text { (MADI; CERRI, } \\
\text { 2017) } \\
\text { Rev. Assoc. Med. } \\
\text { Bras. } \\
\text { Medical oncology, } \\
\text { health services, } \\
\text { health policy, } \\
\begin{array}{l}\text { planning and } \\
\text { management }\end{array}\end{array}$ & $\begin{array}{l}\text { por meio da análise da } \\
\text { estrutura da rede } \\
\text { instalada e habilitada } \\
\text { para tratamento e } \\
\text { suas características } \\
\text { quanto ao perfil e à } \\
\text { distribuição } \\
\text { estabelecimentos, dos } \\
\text { estrutura e serviços } \\
\text { disponíveis e } \\
\text { produção pínima } \\
\text { anual para a } \\
\text { manutenção } \\
\text { excelência. } \\
\text { Estudo de caso único } \\
\text { e integrado, utilizando } \\
\text { dados primários e } \\
\text { secundários. }\end{array}$ & $\begin{array}{l}\text { câncer, porém } \text { havia } \\
\text { diferenças regionais e ampla } \\
\text { variação de produção entre } \\
\text { os serviços, o que } \\
\text { provavelmente impactava no } \\
\text { acesso dos pacientes, } \\
\text { promovia a criação de filas } \\
\text { de espera ao mesmo tempo } \\
\text { que havia serviços com } \\
\text { ociosidade nas instalações. }\end{array}$ \\
\hline E3 & $\begin{array}{l}\text { Estudo de análise } \\
\text { de rede do fluxo de } \\
\text { pacientes de } \\
\text { câncer de mama no } \\
\text { Brasil entre } 2014 \text { e } \\
2016 \text { (SALDANHA, } \\
\text { et. al., 2019) } \\
\text { Cad. Saúde Pública } \\
\text { (Online) } \\
\text { Neoplasias de } \\
\text { Mama; Acesso aos } \\
\text { Serviços de Saúde; } \\
\text { Sistemas de } \\
\text { Informação }\end{array}$ & $\begin{array}{l}\text { Analisar o fluxo de } \\
\text { pacientes oncológicos } \\
\text { de mama que são } \\
\text { atendidos fora de seu } \\
\text { domicílio de } \\
\text { residência. } \\
\text { Considerando a } \\
\text { tipologia de estudos } \\
\text { de redes descrita na } \\
\text { obra de revisão de } \\
\text { Luke \& Harris (2007), } \\
\text { o presente trabalho se } \\
\text { enquadra como um } \\
\text { estudo da "estrutura } \\
\text { interorganizacional de } \\
\text { sistemas de saúde", } \\
\text { com o diferencial da } \\
\text { aplicação de diversas } \\
\text { métricas de } \\
\text { modularidade e da } \\
\text { formação de } \\
\text { comunidades. }\end{array}$ & 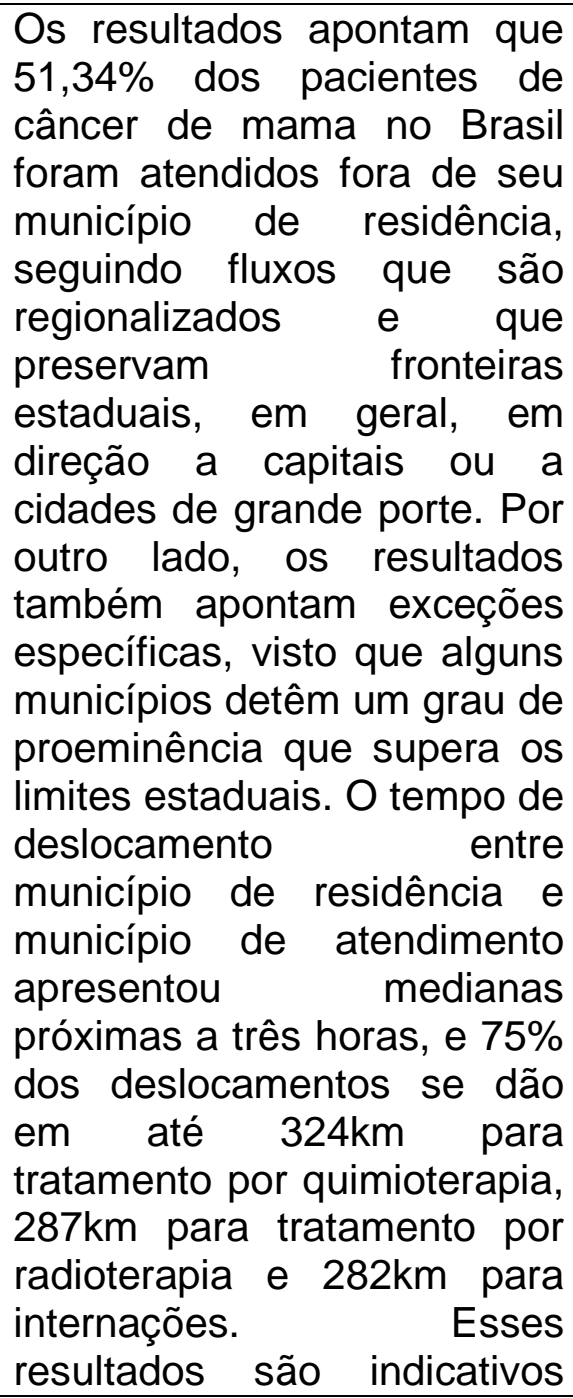 \\
\hline
\end{tabular}




\begin{tabular}{|c|c|c|c|}
\hline N.ㅇ & $\begin{array}{l}\text { REFERÊNCIAS E } \\
\text { DESCRITORES }\end{array}$ & $\begin{array}{l}\text { OBJETIVOS / } \\
\text { MÉTODOS }\end{array}$ & $\begin{array}{l}\text { PRINCIPAIS RESULTADOS } \\
\text { / CONCLUSÕES }\end{array}$ \\
\hline & & & $\begin{array}{l}\text { das dificuldades de acesso } \\
\text { aos serviços de oncologia, o } \\
\text { que potencialmente agrava a } \\
\text { experiência do adoecimento } \\
\text { oncológico em termos de } \\
\text { impacto no indivíduo e em } \\
\text { sua família. }\end{array}$ \\
\hline E4 & $\begin{array}{l}\text { Fluxo assistencial } \\
\text { de usuárias com } \\
\text { câncer de mama na } \\
\text { rede pública de } \\
\text { atenção à saúde } \\
\text { (TOLEDO et. al., } \\
\text { 2016) } \\
\text { Rev. Eletrônica } \\
\text { Enferm. } \\
\text { Neoplasias da } \\
\text { Mama; Acesso aos } \\
\text { Serviços de Saúde; } \\
\text { Serviços de Saúde } \\
\text { da Mulher }\end{array}$ & $\begin{array}{l}\text { Analisar a prevalência } \\
\text { e os fatores } \\
\text { associados ao fluxo } \\
\text { assistencial nos três } \\
\text { níveis de atenção à } \\
\text { saúde exclusivamente } \\
\text { no Sistema Único de } \\
\text { Saúde (SUS) de } \\
\text { usuárias com câncer } \\
\text { de mama. } \\
\text { Estudo transversal } \\
\text { analítico }\end{array}$ & $\begin{array}{l}\text { A prevalência de utilização } \\
\text { do fluxo realizado } \\
\text { exclusivamente no SUS, nos } \\
\text { três níveis de atenção à } \\
\text { saúde, foi de } 43,5 \% \text { (IC95\% } \\
33,6-53,7) \text {. Cerca de um } \\
\text { terço das mulheres realizou } \\
\text { consultas ou exames na rede } \\
\text { privada, principalmente a } \\
\text { mamografia. Os fatores } \\
\text { associados à utilização do } \\
\text { fluxo exclusivamente SUS, } \\
\text { nos três níveis, foi idade } \leq 20 \\
\text { anos na primeira consulta } \\
\text { ginecológica (OR=2,55) e } \\
\text { estar sem trabalho } \\
\text { remunerado (OR=3,28). } \\
\text { Estes resultados sugerem a } \\
\text { necessidade de melhoria de } \\
\text { acesso na rede pública de } \\
\text { atenção à saúde para } \\
\text { mulheres com câncer de } \\
\text { mama. }\end{array}$ \\
\hline E5 & $\begin{array}{l}\text { O caminhor do } \\
\text { diagnósticorar à } \\
\text { reabilitação: os } \\
\text { sentimentos e rede } \\
\text { de apoior das } \\
\text { mulheres que } \\
\text { vivenciam o câncer } \\
\text { e a mastectomia } \\
\text { (URIO et. al., 2019) } \\
\text { Rev. Pesqui. Cuid. } \\
\text { Fundam. (Online) } \\
\text { Saúde da Mulher, } \\
\text { Câncer de mama, } \\
\text { Mastectomia, } \\
\text { Sentimentos }\end{array}$ & 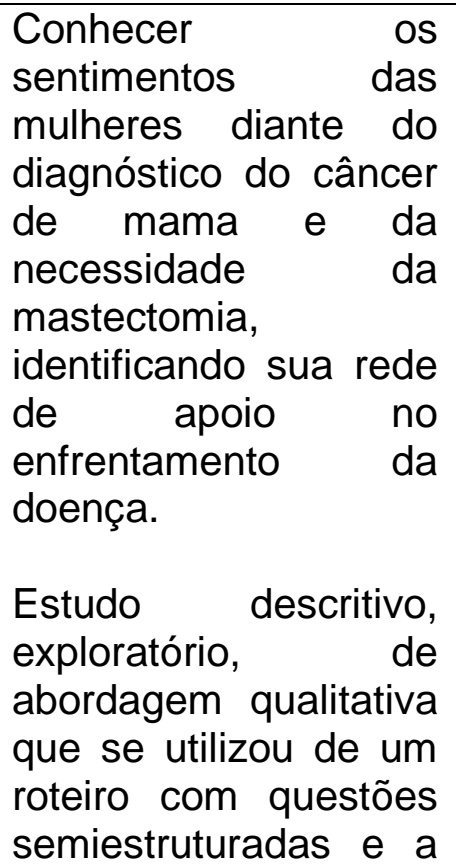 & $\begin{array}{l}\text { Os sentimentos diante do } \\
\text { diagnóstico de câncer vão } \\
\text { desde o desespero, o medo } \\
\text { da morte, até o esforço pela } \\
\text { superação. Quanto ao } \\
\text { sentimento na vivência da } \\
\text { mastectomia surgiu o abalo, } \\
\text { o pensamento negativo, a } \\
\text { compreensão da } \\
\text { necessidade de retirar a } \\
\text { mama e a resiliência. As } \\
\text { redes de apoio foram } \\
\text { evidenciadas pela família, } \\
\text { pelos amigos e pela fé. } \\
\text { Conclui-se que ao se } \\
\text { depararem com } \\
\text { diagnóstico, muitas são as } \\
\text { incertezas da mulher, }\end{array}$ \\
\hline
\end{tabular}




\begin{tabular}{|c|c|c|c|}
\hline N.ㅇ & $\begin{array}{l}\text { REFERÊNCIAS E } \\
\text { DESCRITORES }\end{array}$ & $\begin{array}{l}\text { OBJETIVOS / } \\
\text { MÉTODOS }\end{array}$ & $\begin{array}{l}\text { PRINCIPAIS RESULTADOS } \\
\text { / CONCLUSÕES }\end{array}$ \\
\hline & & $\begin{array}{ll}\text { organização } & \text { dos } \\
\text { dados ocorreu por } & \text { por da análise de } \\
\text { meio da } \\
\text { conteúdo. }\end{array}$ & $\begin{array}{l}\text { tornando-se necessário o } \\
\text { despertar dos profissionais } \\
\text { da saúde em apoiar a mulher } \\
\text { no enfrentamento desta } \\
\text { doença. }\end{array}$ \\
\hline E6 & 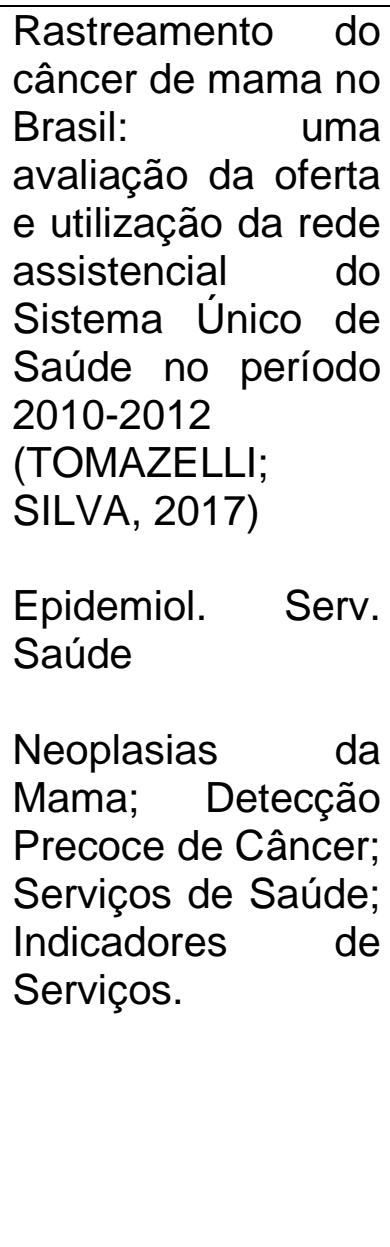 & 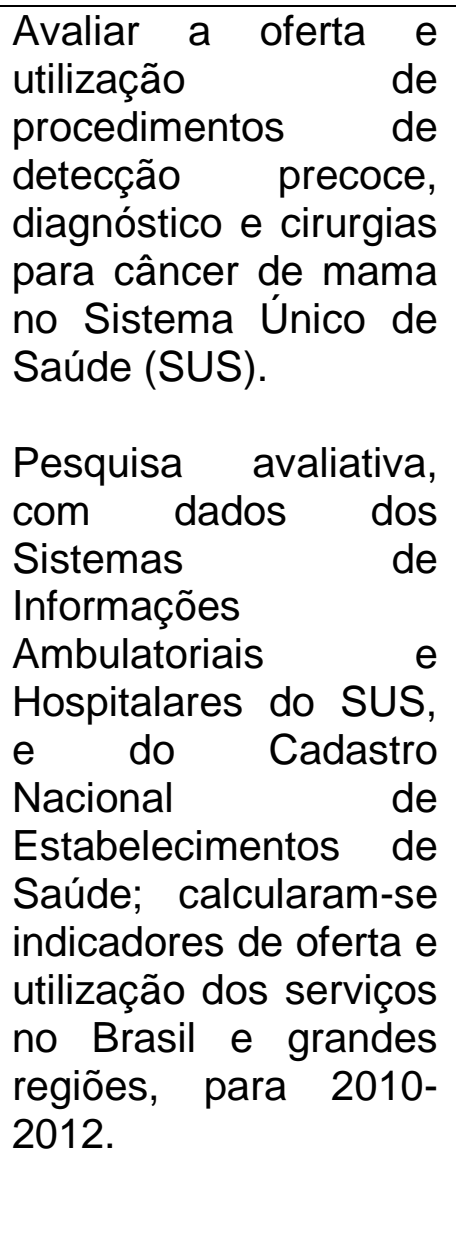 & $\begin{array}{l}\text { A razão entre mamógrafos e } \\
\text { população feminina/100 mil } \\
\text { permaneceu estável }(1,5) \text {, } \\
\text { variando de } 1,0 \text { na região } \\
\text { Norte a } 2,0 \text { na Sul; a razão } \\
\text { entre mamografias e } \\
\text { população feminina/100 mil } \\
\text { aumentou em todas as } \\
\text { regiões; verificou-se baixa } \\
\text { proporção de procedimentos } \\
\text { diagnósticos em relação ao } \\
\text { estimado (biópsia; } 11,5 \% \text {; } \\
\text { punção por agulha grossa, } \\
\text { 16,6\% em mulheres de } 50-69 \\
\text { anos); a capacidade } \\
\text { instalada diminuiu mas a } \\
\text { produção aumentou, e a } \\
\text { oferta disponível (3.995.627) } \\
\text { foi inferior à necessária para } \\
\text { cobertura de } 70 \% \\
\text { (7.785.415). } \\
\text { desigualdades na oferta e } \\
\text { utilização dos procedimentos, } \\
\text { segundo regiões do País, } \\
\text { sendo crítico o acesso ao } \\
\text { diagnóstico. }\end{array}$ \\
\hline E7 & $\begin{array}{l}\text { Social } \\
\text { network of women } \\
\text { with breast cancer / } \\
\text { Rede de apoio } \\
\text { social à mulher } \\
\text { com câncer de } \\
\text { mama (VARGAS } \\
\text { et. al., 2020) } \\
\text { Rev. Pesqui. (Univ. } \\
\text { Fed. Estado Rio J., } \\
\text { Online) } \\
\text { Social support; } \\
\text { Nursing; Nursing } \\
\text { care; Education; } \\
\text { Breast neoplasms }\end{array}$ & $\begin{array}{l}\text { Conhecer a rede de } \\
\text { apoio social à mulher } \\
\text { com câncer de mama, } \\
\text { participante de um } \\
\text { grupo de apoio do Rio } \\
\text { Grande do Sul. } \\
\text { Estudo qualitativo, } \\
\text { exploratório, com } \\
\text { descritivo, } \\
\text { mulheres de um grupo } \\
\text { de apoio }\end{array}$ & 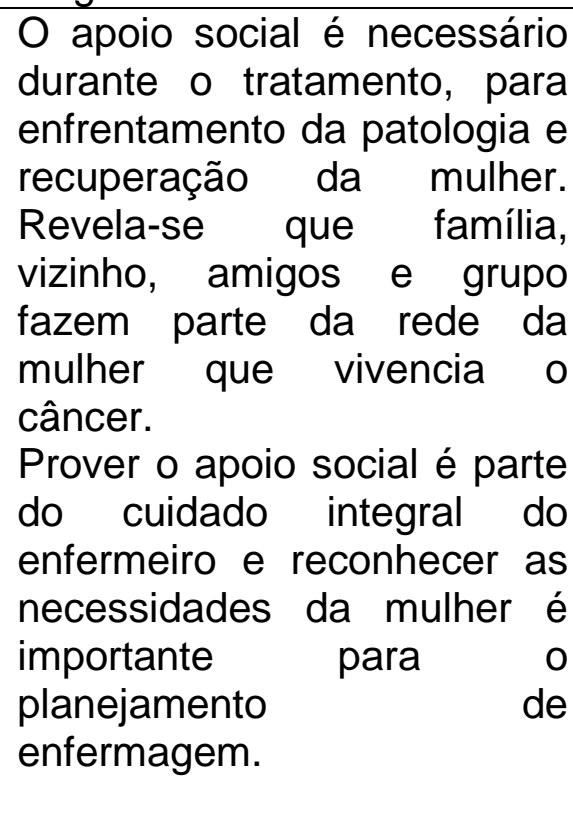 \\
\hline
\end{tabular}




\begin{tabular}{|c|c|c|c|}
\hline N. $\stackrel{-}{ }$ & $\begin{array}{l}\text { REFERÊNCIAS E } \\
\text { DESCRITORES }\end{array}$ & $\begin{array}{l}\text { OBJETIVOS / } \\
\text { MÉTODOS }\end{array}$ & $\begin{array}{l}\text { PRINCIPAIS RESULTADOS } \\
\text { / CONCLUSÕES }\end{array}$ \\
\hline E8 & $\begin{array}{l}\text { Rede social de } \\
\text { apoio do homem } \\
\text { sobrevivente ao } \\
\text { câncer: estudo de } \\
\text { caso etnográfico } \\
\text { (PINTO et. al., } \\
2017) \\
\text { Rev. Pesqui. Cuid. } \\
\text { Fundam. (Online) } \\
\text { Apoio Social; } \\
\text { Saúde do Homem; } \\
\text { Neoplasias da } \\
\text { Próstata }\end{array}$ & $\begin{array}{l}\text { Trata-se de um estudo } \\
\text { de caso etnográfico } \\
\text { realizado com dois } \\
\text { homens sobreviventes } \\
\text { ao câncer de próstata } \\
\text { com alto grau de } \\
\text { resiliência. }\end{array}$ & $\begin{array}{l}\text { Pela análise dos dados, } \\
\text { construíram-se três unidades } \\
\text { de sentido: O papel do } \\
\text { homem no âmbito familiar, A } \\
\text { crença religiosa como forma } \\
\text { de apoio e Relação médico- } \\
\text { paciente implicações na } \\
\text { sobrevivência do homem ao } \\
\text { câncer. Apreende-se que a } \\
\text { família e a espiritualidade } \\
\text { destes homens foram } \\
\text { importantes redes de apoio } \\
\text { social na construção de sua } \\
\text { sobrevivência, bem como } \\
\text { uma comunicação } \\
\text { esclarecedora prestada pelos } \\
\text { profissionais de saúde } \\
\text { auxiliou a vivenciar esse } \\
\text { processo com maior } \\
\text { segurança e tranquilidade. }\end{array}$ \\
\hline E9 & $\begin{array}{l}\text { Rede social no } \\
\text { cuidado à pessoa } \\
\text { estomizada por } \\
\text { câncer colorretal } \\
\text { (NASCENTES et. } \\
\text { al., 2019) } \\
\text { Rev. Enferm. UFPE } \\
\text { on line } \\
\text { Rede Social; Apoio } \\
\text { Social; Neoplasias } \\
\text { Colorretais; } \\
\text { Estomia; Relações } \\
\text { Profissional } \\
\text { Família; } \\
\text { Enfermagem. }\end{array}$ & $\begin{array}{l}\text { Compreender } r \text { a } \\
\text { influência da rede } \\
\text { social no cuidado de } \\
\text { pessoas com estomia } \\
\text { por câncer colorretal e } \\
\text { descrever o tipo de } \\
\text { apoio que a rede } \\
\text { social oferece para } \\
\text { essas pessoas. } \\
\text { Trata-se de um estudo } \\
\text { qualitativo, descritivo, } \\
\text { cujos dados foram } \\
\text { coletados por meio de } \\
\text { entrevista } \\
\text { semiestruturada, } \\
\text { submetendo-os a } \\
\text { técnica de Análise de } \\
\text { Conteúdo. }\end{array}$ & $\begin{array}{l}\text { Observa-se que a rede social } \\
\text { primária foi composta } \\
\text { principalmente por filhos, } \\
\text { irmãos, cônjuges e amigos, } \\
\text { que ofereceram apoio } \\
\text { emocional, material e } \\
\text { cuidados. A rede social } \\
\text { secundária, composta pelos } \\
\text { profissionais de ambulatórios } \\
\text { e hospitais, proporcionou } \\
\text { acolhimento, orientações e } \\
\text { suporte material. Revela-se a } \\
\text { fragilidade das unidades de } \\
\text { atenção primária à saúde } \\
\text { para atender as demandas } \\
\text { de cuidado. }\end{array}$ \\
\hline E10 & $\begin{array}{l}\text { Trajetória de } \\
\text { Mulheres } \\
\text { Rastreadas para o } \\
\text { Câncer de Mama } \\
\text { na Rede Pública de } \\
\text { Saúde } \\
\text { (TOMAZELLI et. al. } \\
\text { 2018) }\end{array}$ & $\begin{array}{lr}\text { Estimar o } & \text { intervalo de } \\
\text { tempo entre } & \text { o } \\
\text { resultado suspeito de } \\
\text { malignidade } & \text { pela } \\
\text { mamografia e o } & \text { início } \\
\text { do } & \text { primeiro } \\
\text { tratamento, } & \text { e } \\
\text { identificar r fatores } \\
\text { associados ao seu }\end{array}$ & $\begin{array}{l}\text { Entre } 158 \text { mulheres com } \\
\text { mamografia alterada, foram } \\
\text { identificados registros de } 66 \\
(41,8 \%) \text { casos de câncer de } \\
\text { mama. Destes, } 12,1 \% \text { tinham } \\
\text { informações sobre biópsias } \\
\text { prévias. O tempo mediano } \\
\text { entre a mamografia e o início } \\
\text { do tratamento foi de } 206\end{array}$ \\
\hline
\end{tabular}




\begin{tabular}{|c|c|c|c|}
\hline N.은 & $\begin{array}{l}\text { REFERÊNCIAS E } \\
\text { DESCRITORES }\end{array}$ & $\begin{array}{l}\text { OBJETIVOS / } \\
\text { MÉTODOS }\end{array}$ & $\begin{array}{l}\text { PRINCIPAIS RESULTADOS } \\
\text { / CONCLUSÕES }\end{array}$ \\
\hline & 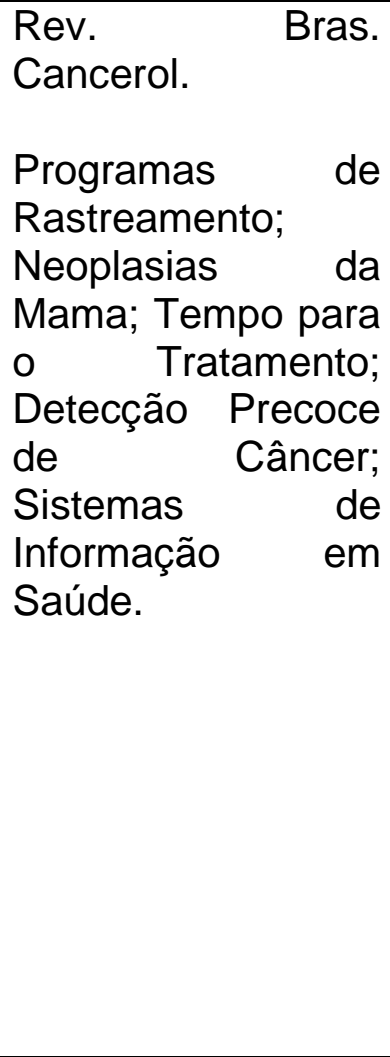 & $\begin{array}{l}\text { início, entre mulheres } \\
\text { rastreadas para } \\
\text { câncer de mama, nos } \\
\text { serviços do Sistema } \\
\text { Único de Saúde (SUS) } \\
\text { do município do Rio } \\
\text { de Janeiro. } \\
\text { Análise descritiva a } \\
\text { partir de relatórios dos } \\
\text { sistemas de } \\
\text { informação. }\end{array}$ & $\begin{array}{l}\text { dias, sendo menor para } \\
\text { mulheres entre } 40-49 \text { anos } \\
\text { (138 dias) do que para as } \\
\text { mais idosas (190 para } \\
\text { mulheres de } 50-59 \text { anos; } 234 \\
\text { dias para mulheres de } 60-69 \\
\text { anos) (Log-rank, p<0,05). } \\
\text { Mulheres que repetiram } \\
\text { mamografia apresentaram } \\
\text { maior atraso (hazard ratio } \\
\text { 0,36; intervalo de confiança } \\
\text { de 95\% 0,19-0,72). Há } \\
\text { poucas biópsias registradas } \\
\text { no SUS e longo tempo até o } \\
\text { início de tratamento, mesmo } \\
\text { quando as mamografias são } \\
\text { solicitadas por hospitais } \\
\text { especializados, } \\
\text { demonstrando necessidade } \\
\text { de o SUS melhorar o } \\
\text { seguimento de mulheres com } \\
\text { mamografia suspeita. }\end{array}$ \\
\hline
\end{tabular}

Fonte: os autores (2021)

A princípio, percebeu-se que os estudos, em grande parte, possuem abordagem qualitativa e são voltados para discutir sobre o câncer de mama, representando $60 \%$ dos estudos analisados, conforme observado na Tabela 2. No que diz respeito ao cuidado prestado ao paciente com câncer, pondera-se que as pesquisas refletem a prática no geral, cujo foco está voltado apenas para os cânceres que possuem maior destaque como política pública e informações alimentadas em sistemas oficiais do Ministério da Saúde (mama e colo do útero). Uma das causas desse distanciamento pode ser a ausência do sistema de informação nacional de que trata 0 Plano de ações estratégicas para o enfrentamento das doenças crônicas não transmissíveis (DCNT) no Brasil 20112022.

TABELA 2 - Descritores usados nas pesquisas relacionadas à rede de atenção à pessoa com câncer. Pau dos Ferros, 2020.

\begin{tabular}{lc}
\multicolumn{1}{c}{ Descritor } & Ocorrências \\
\hline Neoplasias (Câncer) de (da) Mama & 06 \\
Acesso aos Serviços de Saúde & 02 \\
Apoio Social & 02 \\
Detecção Precoce de Câncer & 02 \\
Enfermagem & 02 \\
Saúde da Mulher & 02 \\
Serviços de saúde & 02 \\
Sistemas de Informação (em Saúde) & 02
\end{tabular}




\begin{tabular}{ll} 
Atenção Primária à Saúde & 01 \\
Avaliação em Saúde & 01 \\
Cuidados de enfermagem & 01 \\
Educação & 01 \\
Estomia & 01 \\
Gestão em Saúde & 01 \\
Indicadores de Serviços & 01 \\
Mastectomia & 01 \\
Neoplasias Colorretais & 01 \\
Neoplasias da Próstata & 01 \\
Oncologia médica & 01 \\
Planejamento e gerenciamento & 01 \\
Política de saúde & 01 \\
Programas de Rastreamento & 01 \\
Rede de atenção oncológica & 01 \\
Rede Social & 01 \\
Relações Profissional-Família & 01 \\
Saúde do Homem & 01 \\
Sentimentos & 01 \\
Serviços de Saúde da Mulher & 01 \\
Suporte social & 01 \\
Tempo para o Tratamento & 01 \\
\hline \multicolumn{2}{c}{ Total } \\
\hline
\end{tabular}

Fonte: os autores (2021)

O descritor de maior ocorrência foi sobre o câncer de mama, representando o assunto mais pertinente nos estudos. $O$ acesso aos serviços de saúde, o apoio social, a detecção precoce e a enfermagem são outros descritores encontrados em mais de uma pesquisa. Cabe ainda destacar que os sistemas de informação (em saúde) também foi tema em mais de um estudo, mas que apresenta ainda uma deficiência no que diz respeito à estruturação dos serviços na prática, sendo que o Sistema Único de Saúde (SUS) cobre quase que exclusivamente o acompanhamento dos casos de câncer de mama e colo do útero.

Quanto aos sujeitos pesquisados: quatro estudos (E4, E7, E8, E9) abordaram usuários dos serviços; dois (E2, E5) abrangeram as instituições/serviços assistenciais; outros dois utilizaram dados dos sistemas de informação (E3, E6, E10) e apenas um (E1) tratou com gestores. Isso demonstra que as abordagens estão mais voltadas para os profissionais dos serviços, revelando um certo distanciamento na abordagem sob a percepção dos gestores dos serviços municipais.

No que diz respeito à origem dos indivíduos pesquisados nos estudos, notase que são: quatro da região sudeste (2 SP e 2 RJ); três da região sul (2 RS e 1 $\mathrm{SC})$; um do centro oeste (GO); e dois que abrangem todo o Brasil. Isso demonstra uma concentração das pesquisas sobre a rede de saúde na parte meridional do País, ressaltando a importância de estudos que contemplem a rede de atenção à saúde em outras regiões.

Os descritores mostraram uma variedade de temas associados à temática do câncer, com maior destaque para o de mama, demonstrando a importância e relevância que esta temática tem para o meio científico. Por tratar de uma análise que envolve diversos atores (usuários, gestores, instituições e sistemas de informação), acredita-se que permitiu uma visão ampla sobre a gestão da rede. A 
origem dos estudos, sobretudo da região sudeste, pode estar associada a dois fatores: a presença de programas de pós-graduação direcionados ao estudo da temática ser maior em comparação às demais regiões e/ou revelar uma preocupação mais acentuada sobre a gestão rede de saúde, que deveria se refletir também nas demais regiões do País.

\section{Análise do funcionamento da rede de atenção à saúde}

Quanto à organização da rede de atenção à saúde, classificou-se inicialmente sob a ótica das dimensões da organização do processo de trabalho, elencadas nas diretrizes para o cuidado das pessoas com doenças crônicas nas redes de atenção à saúde e nas linhas de cuidado prioritárias (BRASIL, 2013), conforme apresentado no Quadro 2:

QUADRO 2 - Dimensões de análise dos estudos

\begin{tabular}{|c|c|c|}
\hline DIMENSÕES & ESTUDOS & SITUAÇÕES PERCEBIDAS \\
\hline \multirow{3}{*}{ Acolhimento } & E1 & $\begin{array}{l}\text { Dificuldade no primeiro contato com a Unidade } \\
\text { Básica de Saúde (UBS): filas de espera extensas }\end{array}$ \\
\hline & E5 & $\begin{array}{l}\text { O momento do diagnóstico de câncer representa o } \\
\text { medo da morte }\end{array}$ \\
\hline & E9 & $\begin{array}{l}\text { O acolhimento na unidade hospitalar é importante } \\
\text { para a adesão ao tratamento }\end{array}$ \\
\hline \multirow{5}{*}{$\begin{array}{l}\text { Atenção } \\
\text { centrada na } \\
\text { pessoa e na } \\
\text { família }\end{array}$} & E3 & $\begin{array}{l}\text { Mudança de domicílio em razão da necessidade } \\
\text { de tratamento nos polos mais desenvolvidos }\end{array}$ \\
\hline & E5 & $\begin{array}{l}\text { O apoio familiar é essencial para a recuperação } \\
\text { da saúde e da autoestima }\end{array}$ \\
\hline & E7 & $\begin{array}{l}\text { A família se torna a principal fonte de apoio após o } \\
\text { diagnóstico }\end{array}$ \\
\hline & E8 & $\begin{array}{l}\text { A família representa um vínculo forte na } \\
\text { recuperação }\end{array}$ \\
\hline & E9 & $\begin{array}{l}\text { As relações familiares são apoio essencial para o } \\
\text { cuidado }\end{array}$ \\
\hline \multirow{2}{*}{$\begin{array}{l}\text { Cuidado } \\
\text { continuado / } \\
\text { atenção } \\
\text { programada }\end{array}$} & E1 & $\begin{array}{l}\text { Ausências devido à localidade e à distância da } \\
\text { moradia para o serviço }\end{array}$ \\
\hline & E4 & $\begin{array}{l}\text { Baixa quantidade de usuárias com fluxo } \\
\text { assistencial completamente no SUS nos três } \\
\text { níveis de complexidade }\end{array}$ \\
\hline \multirow{5}{*}{$\begin{array}{l}\text { Atenção } \\
\text { multiprofissional }\end{array}$} & E1 & Alta rotatividade de pessoal \\
\hline & E2 & Falta de profissionais especialistas \\
\hline & E7 & $\begin{array}{l}\text { A equipe de saúde é vista como integrante da } \\
\text { rede de apoio social }\end{array}$ \\
\hline & E8 & $\begin{array}{l}\text { A figura do médico se sobressai em relação aos } \\
\text { outros profissionais }\end{array}$ \\
\hline & E9 & $\begin{array}{l}\text { O ambiente hospitalar é mais bem avaliado que o } \\
\text { da UBS, devido à assistência prestada e à } \\
\text { atenção ao paciente }\end{array}$ \\
\hline $\begin{array}{l}\text { Projeto } \\
\text { terapêutico } \\
\text { singular }\end{array}$ & - & Não foi identificada nenhuma ocorrência \\
\hline $\begin{array}{l}\text { Regulação da } \\
\text { rede de atenção }\end{array}$ & E1 & Deficiência no cumprimento de fluxos e protocolos \\
\hline
\end{tabular}




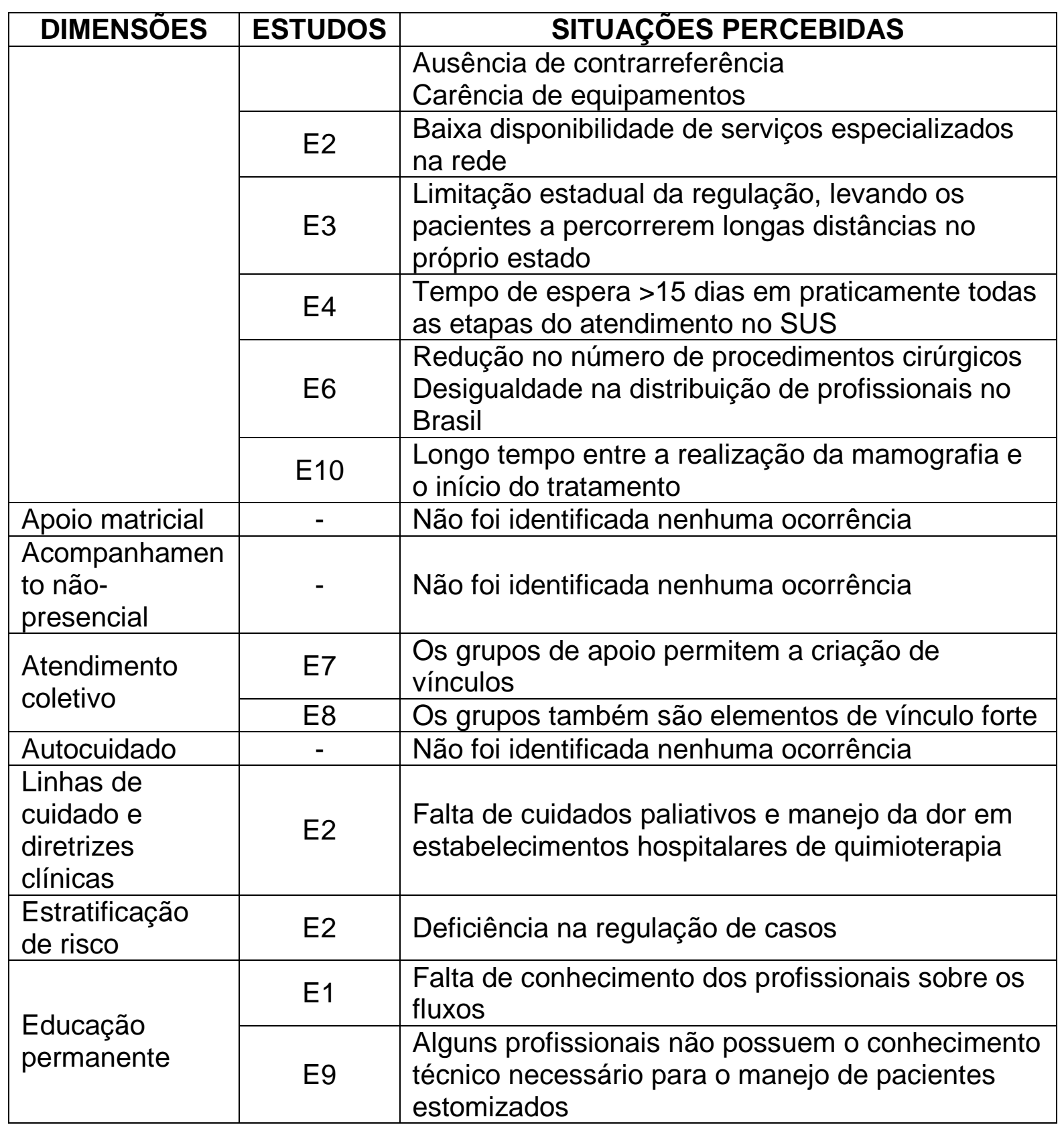

Fonte: os autores (2021)

Das 13 dimensões avaliadas, a "regulação da rede de atenção" foi a mais presente nos artigos, com ocorrência em seis pesquisas, seguida de "atenção multiprofissional" e "atenção centrada na pessoa e na família", com ocorrência em cinco pesquisas cada. Para as dimensões "projeto terapêutico singular", "apoio matricial", "acompanhamento não-presencial" e "autocuidado", não foi identificada nenhuma ocorrência.

A partir dessa classificação inicial, traçaram-se as discussões a partir de três categorias temáticas: Redes de atenção, regulação e níveis de assistência; Rede de apoio familiar, profissional e social; Capacitação profissional para o cuidado.

\section{Redes de atenção, regulação e níveis de assistência}

Esta foi a categoria mais presente nos estudos, tendo em vista que são os pontos cruciais das discussões, sobretudo por envolverem a definição de papeis, responsabilidades e hierarquia da gestão (GOLDMAN et. al., 2019). Ficou 
perceptível a presença de elementos que se complementam e se repetem algumas vezes em alguns dos estudos.

Identificou-se a falta de estruturação na rede (tanto no sistema de referência quanto nos equipamentos de apoio diagnóstico), a insuficiência das linhas de cuidado, diretrizes clínicas e estratificação de risco. Esses fatores refletem a fragilidade do cuidado na atenção especializada, que está caracterizada apenas como ofertante dos serviços ambulatoriais e não um elemento constitutivo da rede de serviços (MADI; CERRI, 2017; GOLDMAN et al., 2019).

Quanto à regulação, observou-se que a redução no número de procedimentos cirúrgicos realizados e a desigualdade na distribuição de profissionais no Brasil compromete muitos elementos da rede de saúde, o que gera filas extensas e deixa deficientes pontos que seriam fundamentais para o cuidado, sobretudo fluxos assistenciais, conforme observado em seis dos estudos (TOLEDO et. al., 2016 ; MADI; CERRI, 2017; TOMAZELLI; SILVA, 2017; TOMAZELLI et al. 2018; GOLDMAN et. al., 2019; SALDANHA, et. al., 2019).

Os que estão relacionados intimamente com a dimensão da regulação, mostraram que há uma relativa satisfação dos pacientes nos prazos dos fluxos exclusivamente no SUS, mas comparando-se com os fluxos que possuem atendimento particular, todos os índices são menos eficientes. Avalia-se ainda uma diversidade nos prazos do tratamento entre pacientes, revelando diferenças de tempo entre exames, diagnóstico e tratamento, demonstrando fragilidades e falta de eficiência na regulação do sistema de saúde para a população (MADI; CERRI, 2017, TOMAZELLI; SILVA, 2017; GOLDMAN et al., 2019).

Percebeu-se ainda relatos de dificuldades no acesso dos pacientes, sobretudo no que diz respeito ao deslocamento dos pacientes e as capacidades de transferir e recebê-los (TOMAZELLI; SILVA, 2017, TOMAZELLI et. al. 2018; SALDANHA, et al., 2019). Isso evidencia principalmente a dimensão da regulação do acesso, que possui limitações tanto pela distância quanto pela disponibilidade dos serviços, levando o paciente muitas vezes a perder o vínculo com seu domicílio devido à necessidade de tratamento em outra região, que implica também no afastamento da rede de apoio familiar (TOMAZELLI et al. 2018; NASCENTES et al., 2019).

\section{Rede de apoio familiar, profissional e social}

A rede de apoio social está relacionada com a atenção multiprofissional, a família e o atendimento coletivo, que permitem a criação de vínculos. Essas são diretrizes essenciais para o fortalecimento da autoestima dos pacientes e para a aceitação da doença e da importância de cuidado. No entanto, isso não dispensa a necessidade de uma atenção integrada com as demais dimensões (PINTO et al., 2017, NASCENTES et al., 2019).

O apoio familiar comprometido com o deslocamento e a possível mudança de domicílio para conseguir o tratamento prejudica duas dimensões: acolhimento e atenção centrada na pessoa e na família. Isso porque a família e os grupos sociais são elementos importantes no processo de recuperação, assim como a equipe de profissionais dos serviços, com destaque para a figura do profissional médico, que geralmente informa o diagnóstico dimensões (PINTO et al., 2017, NASCENTES et al., 2019, VARGAS et al., 2020).

Alguns dos estudos refletem situações e sentimentos que são experimentados por grande parte dos pacientes oncológicos e precisam ser apoiados por outras diretrizes, como o autocuidado, que não aparecem 
caracterizadas nas pesquisas (PINTO et al, 2017; NASCENTES et al., 2019; URIO et al., 2019, VARGAS et al., 2020). Quanto à atenção multiprofissional, o acolhimento nos serviços hospitalares é visto como fator positivo, mas revelam-se dificuldades para manter uma equipe multiprofissional completa (MADI; CERRI, 2017 ; GOLDMAN et al., 2019).

Por fim, os pacientes relatam que os serviços de atenção primária (que deveriam ser ordenadores do cuidado) apresentam deficiências no acolhimento e na assistência, supondo a necessidade de capacitação profissional nas equipes para atuarem como principal referência no cuidado aos pacientes com câncer (TOMAZELLI; SILVA, 2017, PINTO et al., 2017; SALDANHA et al., 2019).

\section{Capacitação profissional para o cuidado}

Mesmo as Unidades Básicas de Saúde sendo consideradas elementos integrantes da rede de apoio social, a capacitação profissional das equipes de atenção primária é avaliada como inferior às hospitalares. Observou-se uma precariedade do acolhimento, associado à falta de conhecimento dos profissionais sobre os fluxos e de conhecimento técnico necessário para o manejo dos pacientes, revelando a ausência de práticas da educação permanente, que seriam essenciais ; para o cuidado integral (PINTO et al., 2017; GOLDMAN et al., 2019; NASCENTES et al., 2019).

\section{CONSIDERAÇÕES FINAIS}

Percebeu-se que existe uma quantidade reduzida de pesquisas que associam a gestão do cuidado ao paciente com câncer nas RAS. Avalia-se que a maioria das pesquisas não aborda na sua integralidade uma análise da rede de atenção, mas apenas a discussão de elementos constitutivos dessa rede.

Por outro lado, julga-se que a atenção ao paciente oncológico ainda está muito longe de se constituir como uma rede de cuidado própria, considerando que é um dos braços da rede temática das Doenças Crônicas, o que, de certa forma, prejudica a sua análise por se tratar de um problema de saúde pública que possui perspectivas de expansão nos próximos anos, conforme as estatísticas apresentadas.

A análise dimensional dos estudos revelou carência na discussão de pontos que são de extrema importância para discutir o cuidado nas redes de atenção à saúde, que são: projeto terapêutico singular, apoio matricial, acompanhamento nãopresencial, autocuidado. Isso demonstra fragilidade na gestão das redes, no sentido de que as avaliações são fragmentadas e os serviços não garantem a existência de fluxos assistenciais efetivos.

Portanto, avaliou-se que o cuidado ao paciente oncológico ainda é desenvolvido de forma fragmentada, devido à irregularidade de oferta dos serviços pela distribuição desigual de profissionais e equipamentos. A ausência de uma rede efetiva, com fluxos definidos e um sistema de regulação tempestivo, associados à limitação da capacitação profissional e a ausência de práticas de educação permanente contribuem para que o cuidado integral aos pacientes não seja garantido.

\section{REFERÊNCIAS}

BRASIL. Ministério da Saúde. Secretaria de Vigilância em Saúde. Departamento de Análise de Situação de Saúde. Plano de ações estratégicas para o 
enfrentamento das doenças crônicas não transmissíveis (DCNT) no Brasil 2011-2022. Brasília: Ministério da Saúde, 2011.

. Ministério da Saúde. Secretaria de Vigilância em Saúde. Departamento de Vigilância de Doenças e Agravos não Transmissíveis e Promoção da Saúde. Saúde Brasil 2018: uma análise de situação de saúde e das doenças e agravos crônicos: desafios e perspectivas. Brasília: Ministério da Saúde, 2019.

. Ministério da Saúde. Secretaria de Atenção à Saúde. Departamento de Atenção Básica. Diretrizes para o cuidado das pessoas com doenças crônicas nas redes de atenção à saúde e nas linhas de cuidado prioritárias. Brasília: Ministério da Saúde, 2013.

GOLDMAN, R. E.; FIGUEIREDO, E. N.; FUSTINONI, S. M.; SOUZA, K. M. J.; ALMEIDA, A. M.; et. al.. Rede de Atenção ao Câncer de Mama: perspectiva de gestores da saúde. Revista Brasileira de Enfermagem, v.72, p. 274-281, Jan.-Fev. 2019. Supl.1. URL: https://www.scielo.br/j/reben/a/c7QzYYsSb8hLTYyDT6YCkpM /?lang=pt. DOI: https://doi.org/10.1590/0034-7167-2017-0479.

INCA. Instituto Nacional de Câncer José Alencar Gomes da Silva. Estimativa 2020: incidência de câncer no Brasil. Rio de Janeiro: INCA, 2019.

MADI, M. R.; CERRI, G. G. Cancer care network: structure analysis of enabled services. Revista da Associação Médica Brasileira, v. 63, n. 10, p. 890-898, Out. 2017. URL: https://www.scielo.br/j/ramb/a/sTH4cHrb8dcst8gDMvBPDwd/ ?lang=en. DOI: https://doi.org/10.1590/1806-9282.63.10.890.

MENDES, E. V. As redes de atenção à saúde. Brasília: Organização PanAmericana da Saúde, 2011.

NASCENTES, C. C.; MOREIRA, M. C.; OLIVEIRA, N. V. D.; PALASSON, R. R.; GHELMAN, L. G. et. al.. Social network in care for the person ostomized due to colorretal cancer. Journal of Nursing UFPE On Line, n.13, p. 1-7, 2019. URL: https://periodicos.ufpe.br/revistas/revistaenfermagem/article/view/239569/32478. DOI: https://doi.org/10.5205/1981-8963.2019.239569.

PINTO, B. K.; MUNIZ, R. M.; AMARAL, D. E. D.; NEVES, F. B.; et. al.. Rede social de apoio do homem sobrevivente ao câncer: estudo de caso etnográfico. Revista de Pesquisa Cuidado é Fundamental, Online, v. 9, n.3, p. 776-785, jul.-set. 2017. URL: http://www.seer.unirio.br/index.php/cuidadofundamental/article/view/5484. DOI: https://doi.org/10.9789/2175-5361.2017.v9i3.776-785.

SALDANHA, R. F.; XAVIER, D. R.; CARNAVALLI, K. M.; LERNER, K.; BARCELLOS, C. Estudo de análise de rede do fluxo de pacientes de câncer de mama no Brasil entre 2014 e 2016. Cadernos de Saúde Pública, v. 35, n. 7: e00090918, jul. 2019. URL: https://www.scielo.br/j/csp/a/XW9YdLCnFpTJn7QnQ3Xr7GP/?lang=pt. DOI: https://doi.org/10.1590/0102-311X00090918.

TOLÊDO, S. R. S.; ALMEIDA, N. A. M.; SOUZA, M. R.; MINAMISAVA, R. JUNIOR, R. F. Fluxo assistencial de usuárias com câncer de mama na rede pública de 
atenção à saúde. Revista Eletrônica de Enfermagem, n. 18 p. 1-9, mar. 2016. URL: https://revistas.ufg.br/fen/article/view/39147. DOI: https://doi.org/10.5216/ ree.v18.39147.

TOMAZELLI, J. G.; SILVA, G. Rastreamento do câncer de mama no Brasil: uma avaliação da oferta e utilização da rede assistencial do Sistema Único de Saúde no período 2010-2012. Epidemiologia e Serviços de Saúde; v. 26, n. 4, p. 713-724, out.-dez. 2017. URL: https://www.scielo.br/j/ress/a/zyjVm7jV7HB8QHmZw6PP74m/ abstract/?lang=pt. DOI: https://doi.org/10.5123/S1679-49742017000400004.

TOMAZELLI, J. G.; SILVA, I. S.; SILVA, G. A. Trajetória de Mulheres Rastreadas para o Câncer de Mama na Rede Pública de Saúde. Revista Brasileira de Cancerologia, v. 64, n. 4, p. 517-526, 2018. URL: https://rbc.inca.gov.br/revista/ index.php/revista/article/view/200. DOI: https://doi.org/10.32635/2176-9745.RBC. 2018v64n4.200.

URIO, A.; SOUZA, J. B.; MANOROV, M. SOARES, R. B. S. O caminho do diagnóstico à reabilitação: os sentimentos e rede de apoio das mulheres que vivenciam o câncer e a mastectomia. Revista Online de Pesquisa Cuidado é Fundamental. v. 11, n.4, p. 1031-1037, jul.-set. 2019. URL: http://www.seer.unirio.br/ index.php/cuidadofundamental/article/view/6862/pdf. DOI: https://doi.org/10.9789/ 2175-5361.2019.v11i4.1031-1037

VARGAS, G. S. FERREIRA, C. L. L.; VACHT, C. L.; DORNELLES, C. S.; SILVEIRA, V. N. et. al.. Social support network of women with breast cancer. Revista Online de Pesquisa Cuidado é Fundamental. n. 12, p. 68-73, jan.-dez. 2020. URL: http://www.seer.unirio.br/cuidadofundamental/article/view/7030. DOI: https://doi.org/10.9789/2175-5361.rpcfo.v12.7030.

WHO 2011. Global status report on noncommunicable diseases 2010. Geneva: World Health Organization, 2011. 